\title{
Potential risk of oral insulin with adjuvant for the prevention of Type I diabetes: a protocol effective in NOD mice may exacerbate disease in BB rats
}

\author{
K. Bellmann ${ }^{1}$, H. Kolb ${ }^{1}$, S. Rastegar ${ }^{2}$, P. Jee ${ }^{2}$, F. W. Scott ${ }^{2}$ \\ ${ }^{1}$ Institute of Diabetes Research at the University of Düsseldorf, Düsseldorf, Germany \\ ${ }^{2}$ Nutrition Research Division, Health Canada, Ottawa, Canada
}

\begin{abstract}
Summary The impact of oral treatment with insulin on disease development was studied in diabetes prone $\mathrm{BB}$ rats. Because of the positive outcome of a prior study in non obese diabetic (NOD) mice, BB rats received insulin in combination with a bacterial adjuvant. Porcine insulin was given orally twice weekly from 35-100 days of age, the E. coli preparation OM-89 was fed on alternate days. Other groups received vehicle, the bacterial adjuvant, or insulin alone. Both insulin containing oral dosing regimens induced a transient non significant delay in diabetes onset. Insulin alone, however did not decrease the final diabetes incidence. Oral dosing with insulin plus adjuvant caused exacerbation of disease development as judged from the decreased survival rate in comparison with the insulin treated group $(p<0.05)$. Intra-islet infiltration also increased $(p<0.005)$ com-
\end{abstract}

pared with the insulin or vehicle treated groups. The effect correlated with enhanced interferon gamma (IFN $\gamma$ ) and decreased interleukin 10 (IL-10) gene expression in the gut suggesting a shift towards proinflammatory Thelper 1 (Th1) reactivity $(p<0.01)$. Although treatment with adjuvant alone also increased the degree of insulitis, an enhanced incidence of diabetes and a shift in cytokine expression was only seen in the group receiving insulin plus adjuvant. Taken together, the data suggest that treatment with a bacterial adjuvant and oral insulin may alter the gut immunoregulatory state such that disease promoting rather than protective immune responses are induced. [Diabetologia (1998) 41: 844-847]

Keywords Oral tolerance, oral insulin, BB rat, immune intervention, gut, cytokines.
Mucosal dosing with autoantigens has been regarded as a benign approach for the treatment of inflammatory diseases of assumed autoimmune origin [1]. The available evidence suggests that giving antigen orally causes apoptosis or anergy or both in specific T cells, or induces antigen reactive $\mathrm{T}$ cells releasing anti-inflammatory cytokines - in particular transforming

Received: 16 December 1997 and in revised form: 4 March 1998

Corresponding author: Dr. H. Kolb, Diabetes Research Institute, Auf'm Hennekamp 65, D-40225 Düsseldorf, Germany Abbreviations: NOD, Non obese diabetic; TGF $\beta$, transforming growth factor beta; IL, interleukin; Th, T helpes; PBS, phosphate buffered saline; RT-PCR, reverse transcriptase polymerase chain reaction; IFN $\gamma$, interferon gamma; iNOS, inducible ND-synthase; BBdp, BioBreeding diabetes prone rats; OVA. growth factor beta TGF $\beta$, interleukin (IL)- 4 or IL-10 - or both. The latter cells are thought to migrate from the gut associated immune system to the sites of organ inflammation and suppress tissue destructive immune reactions [1].

Giving insulin orally or through the nose is presently being tried in clinical trials of diabetes prevention. These studies are based on previous successful attempts to delay and partially prevent disease development in diabetes prone non obese diabetic (NOD) mice [2]. In two other models of human Type I diabetes, the low dose streptozotocin induced diabetes of mice and the diabetes prone BB rat, a beneficial impact of oral insulin dosing on disease development was not observed [3,4].

We recently reported that diabetes prevention by oral insulin in NOD mice could be additionally improved by feeding a bacterial adjuvant on alternate 
days [5]. The possible mechanism involved a shift towards $\mathrm{T}$ helper (Th) 2 cytokine expression in the gut in response to repeated dosing of the adjuvant. Before applying an intervention protocol effective in the NOD mouse to human patients, we tested the approach in the other well characterised model of spontaneous Type I diabetes, the BB rat.

\section{Materials and methods}

Animals. Diabetes prone BB rats were kindly provided by Dr. Pierre Thibert, Animal Resources Division of Health Canada, (Ottawa, Canada), where the stock animals were maintained under specific pathogen-free conditions. Routine serological analyses confirmed bacterial and viral antibody-free status. Animals received a standard, open formula NIH-07 (NIH) diet (Zeigler Brothers, Gardiner, Pa., USA) and tap water ad libitum from weaning at 23 days of age when they were randomised into sex and litter matched control and experimental groups. Animals were dosed orally from 35 days of age with $1 \mathrm{ml}$ of phosphate buffered saline (PBS) (vehicle) or test solution until 100 days of age. Groups of 22-24 rats received either PBS (control), porcine insulin (2 mg per rat, Hoechst AG, Frankfurt, Germany), OM-89 (40 mg active substance/kg body weight, Laboratoires-OM, Geneva, Switzerland) or insulin plus OM-89. Insulin was given twice weekly (Tuesday, Thursday) while OM-89 was given on alternate days (Monday, Wednesday, Friday). OM-89 is an endotoxin-free ( $<12 \mathrm{ppm}$ ) extract of $E$. coli containing mainly acidic glycolipoproteins of 10-300 kDa molecular weight of mostly unknown nature [5]. Wistar rats (50 day old) were provided from the central animal facilities, University of Düsseldorf.

Animals were tested twice weekly for glucosuria and other clinical symptoms of diabetes. In suspected diabetic rats, blood glucose was determined using a glucometer. Diabetes was defined by fasting hyperglycaemia ( $>11.1 \mathrm{mmol}$ glucose per $\mathrm{ml}$ ) with concomitant weight loss. At 80 days of age, 6 animals from each group were killed by exsanguination under $3 \%$ isoflurane anesthesia. For histological analysis, the pancreas was removed from each rat and fixed in Bouin's solution. The proximal part of the gut was dissected, flushed with ice-cold PBS and a 4-5 cm long piece of the intestine was used for RNA extraction. Additional rats (Wistar and $\mathrm{BB}$ ) at 50 days of age were killed for RNA isolation of the gut. The "Principles of laboratory animal care" (NIH publication No. 85-23, revised 1985) were followed, as well as the current version of the German Law on the Protection of Animals.

Histological analysis. Pancreata were removed, cleaned of fat and lymph nodes and fixed in Bouin's solution. Sections $(5 \mu \mathrm{m})$ were cut at $100 \mu \mathrm{m}$ intervals and stained with haematoxylin and eosin for evaluation of macrophage/mononuclear cell infiltration (insulitis).

All islets in two separate sections at least $100 \mu \mathrm{m}$ apart were evaluated. A subjective rating was given for the extent of insulitis. Peri-insulitis was defined as 5 mononuclear cells or more surrounding the islet, and intrainsulitis as 5 mononuclear cells or more inside the boundary of the islet.

$m R N A$ analysis. Total RNA was isolated from the small intestine using Trizol Reagent (Gibco BRL, Berlin, Germany). We determined and quantified specific mRNA by reverse transcriptase polymerase chain reaction (RT-PCR) as described elsewhere [6]. We did control experiments to check for a linear

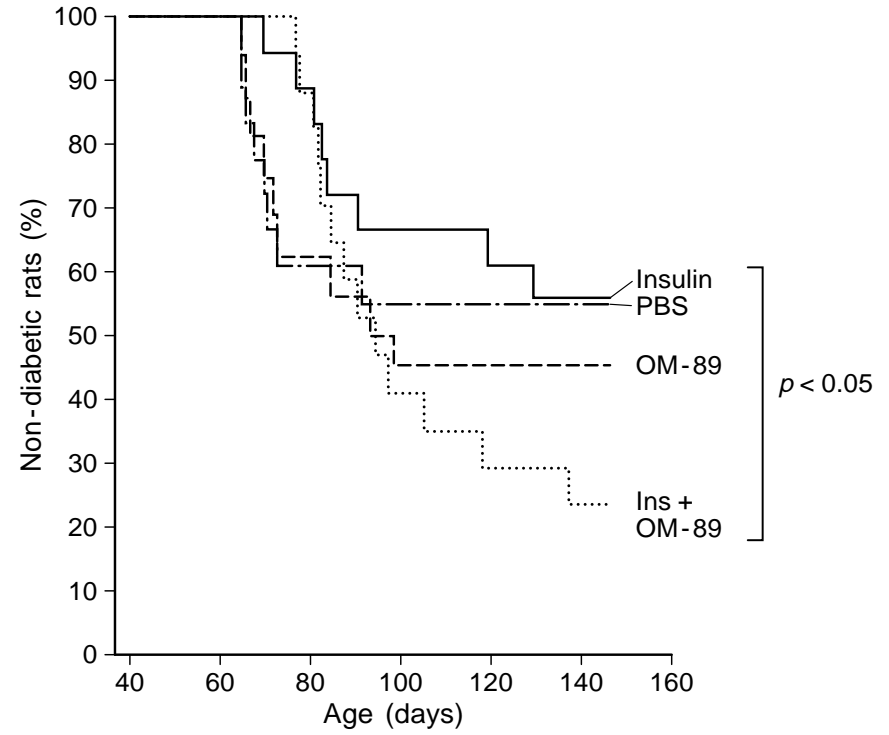

Fig. 1. Impact of oral insulin and bacterial adjuvant on diabetes incidence. BBdp rats received oral doses of vehicle (PBS; $n=18$ ), insulin (INS; $n=18$ ), the bacterial preparation OM89 (OM; $n=16)$, or a combination of insulin and OM-89 (INS $+\mathrm{OM} ; n=17)$ between 35 and 100 days of age. $P<0.05$ between the insulin and the insulin and OM-89 treated groups

relationship between amount of PCR product and amplification cycle number. Specific primers were used for $\beta$-actin, interferon gamma (IFN $\gamma$ ), IL-10, (Clontech Laboratories Inc., Palo Alto, Calif., USA) and inducible NO-synthase (iNOS) [6]. PCR products were labelled by hybridization to ${ }^{32} \mathrm{P}$ labelled probes binding at sites between the primer sequences. Signals were quantified by measuring the ${ }^{32} \mathrm{P}$ stimulated luminescence (PSL) using a phosphoimager. Relative PSL was calculated by normalization of the measured PSL to the strength of the $\beta$-actin signal, which was assigned a value of one.

Statistics. Differences between mean insulitis scores were evaluated using a one way analysis of variance (ANOVA) with posthoc comparison by the (LSD) method. Differences in diabetes development were analysed using survival analysis with log rank test, differences in cytokine mRNA levels were analysed using two-sided Student's t-test, with the level of significance set as $p$ less than 0.05 .

\section{Results}

In vehicle-treated BioBreeding diabetes prone (BBdp) rats, the first cases of diabetes were seen in animals between 60 and 70 days of age. The incidence of diabetes reached $44 \%$ by 100 days, with no further increase up to age 146 days (Fig. 1). Bacterial adjuvant, OM-89, given orally did not alter the kinetics of disease development. Interestingly, both insulin containing treatment regimens showed an initial but transient delay of diabetes onset of 1-2 weeks. The insulin-dosed group finally reached the same incidence as the control group (44\%, Fig. 1). Surprisingly, the diabetes incidence steadily increased in the group receiving insulin plus adjuvant, reaching $76 \%$ 
Table 1. Analysis of islet infiltration

\begin{tabular}{lllll}
\hline $\begin{array}{l}\text { Oral } \\
\text { treatment }\end{array}$ & $\begin{array}{l}\text { Number } \\
\text { of rats }\end{array}$ & $\begin{array}{l}\text { Total num- } \\
\text { ber of islets }\end{array}$ & $\begin{array}{l}\text { \% Islets with } \\
\text { peri-insulitis }\end{array}$ & $\begin{array}{l}\text { \% Islets with } \\
\text { intra-insulitis }\end{array}$ \\
\hline PBS & 6 & 408 & 11 & 48 \\
Insulin & 6 & 360 & 10 & 55 \\
OM-89 & 6 & 324 & 8 & $\mathbf{7 9}$ \\
Ins + OM-89 & 6 & 432 & 8 & $\mathbf{9 0}$
\end{tabular}

Each islet was classified as normal, with peri-insulitis or intrainsulitis. Bold type denotes change to the control group of $p<0.005$.

at the end of the observation period (Fig. 1). The increased rate of diabetes development was significant in comparison with the group receiving insulin alone $(p<0.05)$ but not when compared with the vehicle treated group $(p=0.16)$.

Analysis of islet histology was performed in diabetes prone $\mathrm{BB}$ rats at a crucial prediabetic stage, e. $g$. 80 days of age, when large numbers of rats became diabetic, suggesting peak inflammatory activity in the islets. Rats receiving insulin exhibited a similar percentage of islets with intrainsulitis as in the control group (Table 1). When insulin was given in conjunction with OM-89, however, or OM-89 was given alone, a significant increase of intraislet infiltration was noted (Table $1, p<0.005$ ).

Total gut RNA was analysed by RT-PCR for levels of IFN $\gamma$, IL-10 and iNOS mRNA. Both, IFN $\gamma$ and IL-10, mRNA were present in BBdp rats dosed orally with PBS (Fig. 2 A,B). Oral insulin treatment almost completely down regulated gene expression for both, IFN $\gamma$ and IL-10 $(p<0.05)$. Conversely, treatment with insulin plus adjuvant or adjuvant alone induced a rise of IFN $\gamma$ gene expression $(p<0.05)$ and IL-10 mRNA remained in the range of the control group. As a consequence, the ratio of IFN $\gamma$ to IL-10 was increased, in particular for the group receiving insulin plus OM-89, in comparison with the two other groups (Fig. 2C).

As an additional marker of the gut inflammtory bias we analysed the IFN $\gamma$ to IL- 10 ratio in BB rats at the age of 50 days prior to visible islet infiltration and compared this with genetically closely related Wistar rats of the same age. As shown in Figure 2D we observed a significantly higher IFN $\gamma$ to Il-10 ratio in the $\mathrm{BB}$ rat gut compared with the Wistar rats.

\section{Discussion}

Kinetics of diabetes development in the vehicletreated control group showed that most diabetes cases occurred between 65 and 90 days of age, as observed previously for the Ottawa colony of BB rats [6]. Although diabetes development reached a plateau by 100 days of age in the PBS-treated group,
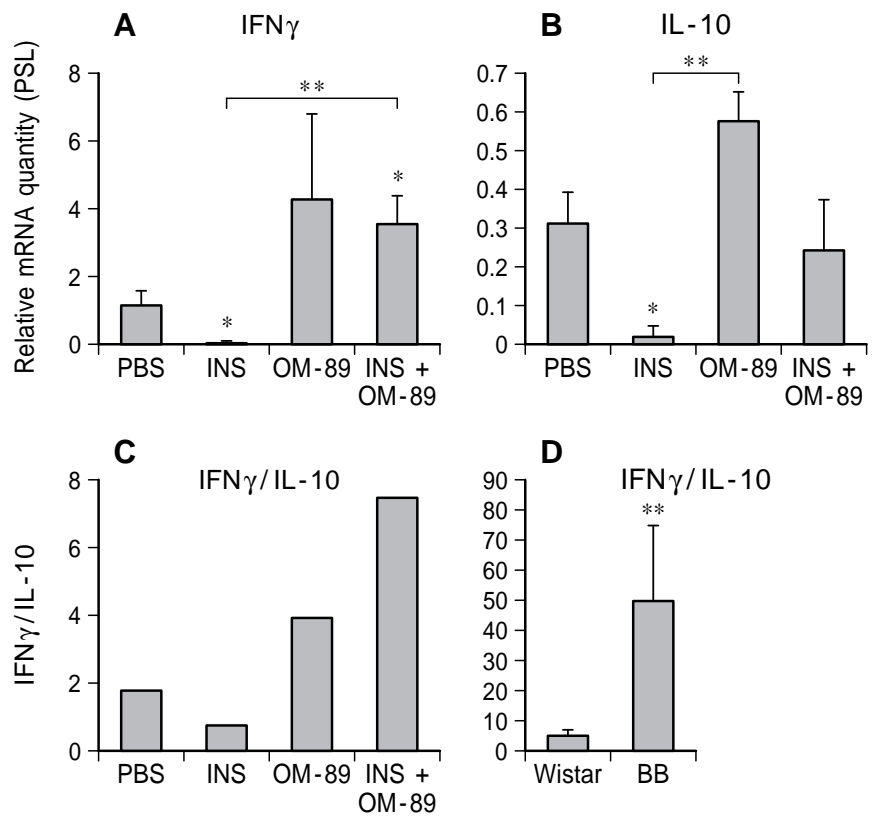

Fig.2A-D. Cytokine gene expression in the gut. Gut RNA was analysed for (A) interferon gamma, and (B) IL-10 by RTPCR with quantitation of PCR-products by posphoimaging. The levels of mRNA were calibrated to the amount of $\beta$-actin mRNA which was set as 1 . The ratio of IFN $\gamma$ vs IL-10 mRNA (C) was calculated from these figures. $\mathbf{A}$ and $\mathbf{B}$ show means \pm SD for gut RNA pooled from six animals per group analysed in three separate experiments. $\mathbf{C}$ shows the quotient of means of $\mathbf{A}$ and $\mathbf{B}$. D shows means \pm SD with $n=3-6$. $*, p<0.05$; **, $p<0.01$ compared with the control if not indicated differently

there was a steady further increase of diabetes cases in the group receiving insulin plus OM-89. Survival analysis showed that the diabetes rate was higher in the group dosed with insulin plus adjuvant compared with insulin alone $(p<0.05)$, suggesting exacerbation of disease.

Both groups receiving insulin showed an initial delay in appearance of diabetes cases. Though the diabetes incidence reached $39 \%$ by 75 days of age in the control group, there were still no cases of diabetes in the group receiving insulin plus OM-89, and less than $10 \%$ of rats became diabetic if insulin alone was given. Although not substantial, the delay was seen only for the two groups receiving autoantigen. The latter result confirms a previous study in which a protective effect of oral insulin in the BB rat was not observed [4]. Delay followed by exacerbation has also been reported in attempts to prevent the development of multiple sclerosis in marmosets by autoantigen treatment [7], and also when low doses of myelin basic protein were fed prior to the induction of experimental allergic encephalomyelitis in mice [8].

The analysis of insulitis in the four groups mirrored the differences observed in rates of diabetes. Oral dosing with insulin or vehicle led to a similar de- 
gree of islet infiltration. There was a non-significant increase of insulitis scores in the group receiving adjuvant alone and significant exacerbation of insulitis in the group dosed with insulin plus adjuvant. In this case, $90 \%$ of islets exhibited intra-islet infiltration, in comparison with $48 \%$ in the PBS-treated group $(p<0.001)$. We observed previously a close correlation between intra-insulitis and a bias towards Th1 cytokine expression in the pancreas [5]. The group receiving insulin plus adjuvant differed from the group dosed with adjuvant alone also with regard to gut cytokine patterns. A shift towards enhanced IFN $\gamma$ over IL-10 production was only seen for the cohort treated with insulin plus OM-89.

The outcome of our study in BB rats contrasted with the result of a similar oral immunisation protocol in NOD mice where dosing with insulin and bacterial adjuvant decreased diabetes incidence and ameliorated islet inflammation [5]. In NOD mice, treatment with insulin and OM-89 shifted the immunoregulatory balance of the gut towards enhanced Th2 cytokine expression [5], which is known to promote oral tolerance responses $[1,2]$. The present findings in the $\mathrm{BB}$ rat gut demonstrate an opposite effect of adding bacterial adjuvant to oral insulin treatment, that is, an increase of Th1 over Th2 reactivity. We chose IFN $\gamma$ and IL-10 for analysis because these two cytokines represent major antagonistic cytokines determining the Th1/Th2 balance [5]. The BB rat gut immune system may have a predisposition towards Th1 reactivity. When comparing the small intestine of untreated $\mathrm{BB}$ rats with that of non-diabetes prone but genetically related Wistar rats a significant bias towards IFN $\gamma$ expression was noted.

A more marked diabetic development by oral ovalbumin has been described for an animal model comprising a transgenic mouse expressing (OVA) on beta cells and carrying an enriched population of OVA-specific $\mathrm{CD}^{+} \mathrm{T}$ cells [9]. This adverse outcome was seen though in a highly artificial animal model where the target autoantigen (OVA) was introduced as a transgene. Furthermore, the immune system in these mice was chimeric and contained a major fraction of $\mathrm{CD}^{+} \mathrm{T}$ cells expressing a transgenic T cell receptor recognising OVA in the context of MHC class I [9]. Our study of BB rats indicates that exacerbation of disease development can also be induced in a spontaneous model of autoimmune diabetes, by oral exposure of susceptible individuals to a natural autoantigen, insulin. We have shown previously that certain wheat or soy proteins of rat laboratory diets are diabetes promoting in BBdp rats, while a hypoallergenic, hydrolysed, protein-based formula is not [10]. Previous studies have shown that bacterial immunostimulatory compounds such as LPS potentiate oral tolerance mechanisms [1]. These studies were performed, however, in normal animals. In this study a comparison with genetically related Wistar rats showed a Th1 bias of cytokine gene expression in the gut of $\mathrm{BB}$ rats. Therefore we assume that the gut immune system of $\mathrm{BB}$ rats is biased towards inflammatory Th1 reactivity in contrast to NOD mice and exhibits impaired oral tolerance. The present findings further underline an important role for the gut in the pathogenesis of Type I diabetes.

In conclusion, our findings in the BBdp rat indicate some potential for untoward effects when insulin is given in the presence of immunostimulatory antigens which could be part of food.

Acknowledgements. We are grateful to Dr. Seipke for porcine insulin and Dr. Farine for OM-89. We thank W. Fingberg and D. Piras for typing the manuscript and Mr. J Souligny in carrying out the animal studies. This work was supported by a grant from the Laboratoires OM, Geneva, Switzerland, the Bundesminister für Gesundheit, the Bundesminister für Bildung, Wissenschaft und Forschung und Technologie and by the Minister für Wissenschaft und Forschung des Landes Nordrhein-Westfalen and in part by a grant from the Medical Research Council of Canada.

\section{References}

1. Weiner HL, Friedmann A, Miller A et al. (1994) Oral tolerance: immunologic mechanisms and treatment of animal and human organspecific autoimmune diseases by oral administration of autoantigens. Annu Rev Immunol 12: 809-837

2. Zhang ZJ, Davidson L, Eisenbarth G, Weiner HL (1991) Suppression of diabetes in nonobese diabetic mice by oral administration of porcine insulin. Proc Natl Acad Sci USA 88: $10252-10256$

3. Kolb H, Zielasek J, Berschick P, Kiesel U (1991) Oral prevention of type 1 diabetes. Autoimmunity 11: 133 (letter)

4. Mordes JP, Schirf B, Roipko D et al. (1996) Oral insulin does not prevent insulin-dependent diabetes mellitus in BB rats. Ann NY Acad Sci 778: 418-421

5. Hartmann B, Bellmann K, Ghiea I, Kleemann R, Kolb H (1997) Oral insulin for diabetes prevention in non-obese mice: potentiation by enhancing Th2 cytokine expression in the gut through bacterial adjuvant. Diabetologia 40: 902-909

7. Genain CP, Abel K, Belmar N et al. (1996) Late complications of immune deviation therapy in a nonhuman primate. Science 274: 2054-2056

8. Meyer AL, Benson JM, Gienapp IE, Cox KL, Whitacre CC (1996) Suppression of murine chronic relapsing experimental autoimmune encephalomyelitis by the oral administration of myelin basic protein. J Immunol 157: 4230-4238

9. Blanas E, Carbone FR, Allison J, Miller JFAP, Heath WR (1996) Induction of autoimmune diabetes by oral administration of autoantigen. Science 274: 1707-1709

10. Scott FW, Cloutier HE, Kleemann R et al. (1997) Potential mechanisms by which certain foods promote or inhibit the development of spontaneous diabetes in BB rats: dose, timing, early effect in islet area, and switch in infiltrate from Th1 to Th2 cells. Diabetes 46: 589-598 